\title{
A Prediction of Blood Flow through a Bypass Graft Using Statistical Methods
}

\author{
Jana Vrbková ${ }^{1}$, Vilém Bruk ${ }^{2}$ \\ 1. Department of Mathematical Analysis and Applications of Mathematics, Faculty of Natural \\ Sciences, Palacky University Olomouc, Czech Republic, \\ 2. Clinic of Cardiosurgery, Faculty Hospital Olomouc, Czech Republic
}

Myocardial revascularization belongs among the most frequent cardiosurgery operations. Perioperative and longterm survival depend on the patency of the graft used and the anastomotic quality. Haemodynamical characteristics measured during a coronary artery bypass graft (CABG) surgery help verify anastomotic quality and also affect longterm graft patency. During CABG surgery (on a heart bypass machine with extracorporal circulation), a surgeon measures blood flow through the bypass at the time the cross clamp is applied to the ascending aorta (blood is not flowing through coronary vessels, rather through the bypass) and later at the same place after removal of the cross clamp. The aim of this article is to find a statistical model for prediction of blood flow through the bypass after removal of the cross clamp based on the blood flow value when the cross clamp is placed on the aorta. When this prediction is good, we will be able to decrease a number of measurements with keeping whole information about an object.

Keywords: myocardial revascularization, prediction of blood flow and blood pressure, multiple regression, nonlinear regression model, linearization, linear regression model with constraints, outlier, leverage

\section{Introduction}

Coronary revascularization belongs among the most frequent cardiosurgery operations in the world as well as in our republic (in the year 2002, 10797 cardiosurgery operations were performed of which 7051 were CABG surgeries) [1]. Perioperative and longterm survival depend on the patency of the graft used and the anastomotic quality. Many authors were concerned with finding characteristics which determine graft patency and anastomotic quality from both a short-term and a long-term point of view. As it turns out, haemodynamical characteristics help to verify anastomotic quality as well as longterm graft patency [2], [3], [4], [5], [6]. Louagie indicates resistance as a dominant characteristic for longterm graft patency. Resistance can be calculated as blood pressure divided by blood flow through a vessel [7]. Although this formula is only a simplification of the reality (blood flow is not a steady flow and blood is not a Newtonian fluid) [8], it is reasonable to think about blood pressure and blood flow as characteristics which determine resistance. Hata [9] confirmed on a set of patients with low free blood flow through an arterial bypass that a left mammary artery (LIMA) is able to adapt its diameter in relation to storage demands of a target coronary bed. It is also known that a flow through a bypass is affected by the competitive flow of a native coronary bed. Furthermore, flow through a bypass is speculated to affect changes of graft patency in relation to a competitive flow and the sensitivity of different kind of bypass 
grafts (LIMA, RIMA, SVG) [10], [11], [12]. During myocardial revascularization (CABG on a heart bypass machine), various measurements of blood flow through the bypass are taken: free-flow (the bypass is not anastomosed on a target coronary bed yet), before removal of the clamp from the aorta (a coronary bed is stored only by bypass - there is no competitive flow) and after removal of the cross clamp from the aorta (with a competitive flow). Sometimes, a surgeon takes two measurements before removal of the clamp from the aorta: one before removal of clamps from other bypasses and one after removal of these clamps (blood flow through a bypass also depends on collaterals). A flowmeter (produced by the Norwegian company Medi-Stim) is used for the measurement of blood flow during aortocoronary bypass surgery at the Faculty Hospital in Olomouc. This machine works by the so called Transit-Time method. The principle is based on the fact that the time required for the ultrasound to pass through blood is slightly longer when it is passing upstream than downstream [13]. A surgeon can observe perioperatively with the aid of this machine the blood flow curve, the blood flow value, the mean flow, the Pulsatility Index [PI=(max. flow - min. flow)/mean flow] and other characteristics. It is possible to connect to this machine two flow probes, two pressure inputs and two auxiliary devices, for example ECG input. The flowmeter can calculate many characteristics from recorded data, for example the Fast Fourier Analysis for a saved curve (of blood flow, pressure etc.). In a small group of patients (35), mean arterial pressure and blood flow through a bypass (a left mammary artery grafted to the left anterior descending artery - LIMA-LAD) were measured during CABG surgery at the time when the cross clamp is applied to the aorta (blood is flowing to a coronary bed only through a bypass) and also at the time when the clamp is removed (a competitive flow). The aim of this article is to find a statistical model for the prediction of blood flow through a bypass after removal of the cross clamp from the aorta based on the blood flow value with the clamp still in the place on the aorta. When this prediction is good, it would be able to decrease a number of measurements with keeping whole information about an object.

\section{Input data and model}

\subsection{Input data}

Data representing blood flow through a bypass (LIMA-LAD) are used for the analysis. The measurements were taken two times: with the cross clamp on the aorta (LIMA-LAD I) and after the clamp was removed (LIMA-LAD III). At the same time, mean arterial pressure was recorded in $\mathrm{mmHg}$ and the flow in $\mathrm{ml} / \mathrm{min}$. Data were obtained from 35 patients during LIMA or BIMA (the measurements were taken on the left mammary artery) CABG surgeries.

Data obtained by measurement at the Clinic of Cardiosurgery, Faculty Hospital in Olomouc are organized in a vector of input data $\zeta=\left(x_{1}, y_{1}, \ldots, x_{n}, y_{n}, \xi_{1}, \eta_{1}, \ldots, \xi_{n}, \eta_{n}\right)$ ' (' signs vector transposition), where

$$
\begin{gathered}
x_{1}, x_{2}, \ldots, x_{n} \\
y_{1}, y_{2}, \ldots, y_{n} \\
\xi_{1}, \xi_{2}, \ldots, \xi_{n} \\
\eta_{1}, \eta_{2}, \ldots, \eta_{n}
\end{gathered}
$$

is blood flow through a bypass LIMA-LAD I,
is mean arterial blood pressure LIMA-LAD I,
is blood flow through a bypass LIMA-LAD III,
is mean arterial blood pressure LIMA-LAD III. 
Table 1. Input data.

\begin{tabular}{|l|l|l|l|l|}
\hline $\mathrm{i}$ & $\mathrm{x}_{\mathrm{i}}$ & $\mathrm{y}_{\mathrm{i}}$ & $\xi_{\mathrm{i}}$ & $\eta_{\mathrm{i}}$ \\
\hline 1 & 80 & 67 & 67 & 65 \\
\hline 2 & 101 & 47 & 40 & 47 \\
\hline 3 & 94 & 68 & 66 & 66 \\
\hline 4 & 53 & 63 & 56 & 63 \\
\hline 5 & 46 & 66 & 41 & 66 \\
\hline 6 & 30 & 63 & 22 & 67 \\
\hline 7 & 34 & 65 & 11 & 64 \\
\hline 8 & 39 & 60 & 42 & 60 \\
\hline 9 & 38 & 77 & 58 & 65 \\
\hline 10 & 55 & 60 & 36 & 55 \\
\hline 11 & 55 & 63 & 35 & 67 \\
\hline 12 & 31 & 60 & 24 & 57 \\
\hline 13 & 85 & 67 & 38 & 65 \\
\hline 14 & 33 & 80 & 15 & 77 \\
\hline 15 & 36 & 70 & 14 & 70 \\
\hline 16 & 74 & 75 & 55 & 65 \\
\hline 17 & 105 & 72 & 98 & 78 \\
\hline
\end{tabular}

\begin{tabular}{|l|l|l|l|l|}
\hline 18 & 26 & 50 & 15 & 54 \\
\hline 19 & 51 & 62 & 38 & 74 \\
\hline 20 & 29 & 77 & 27 & 77 \\
\hline 21 & 84 & 60 & 79 & 67 \\
\hline 22 & 40 & 70 & 49 & 69 \\
\hline 23 & 75 & 65 & 72 & 60 \\
\hline 24 & 24 & 60 & 12 & 56 \\
\hline 25 & 44 & 77 & 24 & 75 \\
\hline 26 & 56 & 62 & 29 & 54 \\
\hline 27 & 38 & 60 & 8 & 52 \\
\hline 28 & 10 & 67 & 2 & 63 \\
\hline 29 & 60 & 65 & 46 & 60 \\
\hline 30 & 30 & 54 & 13 & 47 \\
\hline 31 & 99 & 59 & 96 & 66 \\
\hline 32 & 44 & 76 & 33 & 74 \\
\hline 33 & 36 & 65 & 21 & 57 \\
\hline 34 & 31 & 57 & 22 & 60 \\
\hline 35 & 43 & 76 & 35 & 77 \\
\hline
\end{tabular}

\subsection{Statistical model}

The model of the relationship between blood flow and pressure at the time when the clamp is applied to the aorta (LIMA-LAD I) and at the time when the clamp is removed (LIMA-LAD III) can be assumed in a few forms. The simplest possible form is the classical linear regression model.

$\left(\begin{array}{c}v_{i, 1} \\ v_{i, 2}\end{array}\right) \square\left(\begin{array}{c}\square_{1} \\ \square_{2}\end{array}\right) \square\left(\begin{array}{cc}\square_{11} & \square_{12} \\ \square_{21} & \square_{22}\end{array}\right)\left(\begin{array}{c}x_{i} \\ y_{i}\end{array}\right)$,

where $x_{i}$ and $y_{i}$ are values of the patient's blood flow and blood pressure at the time when the cross clamp is applied to the aorta and $v_{i, 1}$ and $v_{i, 2}$ are predicted values of these parameters after removal of the cross clamp. There are random errors only on the left side in $v_{i, 1}$ and $v_{i, 2}$ values in this model. Parameters $\alpha_{1}, \alpha_{2}, \beta_{11}, \beta_{12}, \beta_{21}, \beta_{22}$ are estimated by the least square method.

However, the presumption of errorless values $x_{i}$ and $y_{i}$ don't correspond to the reality. The "Crystal ball" model, in which we assumed values $x_{i}$ and $y_{i}$ as realizations of random variables, is better. 
$E(\zeta)\left[\square\left[\square_{1,1}, \square_{1,2}, \ldots, \square_{n, 1}, \square_{n, 2}, \square_{1,1}, \square_{1,2}, \ldots, \square_{n, 1}, \square_{n, 2}\left[, \operatorname{Var}(\zeta) \square \square^{2}\left(\begin{array}{cc}2 n, \boldsymbol{\theta} & \mathbf{I} \\ & 2 n, 2 n\end{array}\right)\right.\right.\right.$,

where $\mathrm{E}(\zeta)$ satisfies the conditions:

$$
\left(\begin{array}{l}
E\left(\xi_{i}\right) \\
E\left(\eta_{i}\right)
\end{array}\right)=\left(\begin{array}{l}
\beta_{1} \\
\beta_{2}
\end{array}\right)+\left(\begin{array}{ll}
\beta_{3} & \beta_{4} \\
\beta_{5} & \beta_{6}
\end{array}\right)\left(\begin{array}{l}
E\left(x_{i}\right) \\
E\left(y_{i}\right)
\end{array}\right), i=1,2, \ldots, n
$$

Note: $\mathrm{E}(\zeta)$ and $\operatorname{Var}(\zeta)$ denote the mean value and the variance of the random vector $\xi$.

The variance matrix $\operatorname{Var}(\zeta)$ is assumed in the simplest possible form and it is used for less complexity of following calculations. It will be necessary to assume more complex form of this matrix in further research but at the same time it will lead to a problem of estimation of variance components.

\section{Prediction of blood flow and pressure}

\subsection{The classical regression model}

Estimations of parameters $\alpha_{1}, \alpha_{2}, \beta_{11}, \beta_{12}, \beta_{21}, \beta_{22}$, which we obtain for our data set with usage of function $\operatorname{lm}(\mathrm{y} \sim \mathrm{x})$ (available in software $\mathrm{R})$, are shown in the table 2 .

Table 2. Estimated parameters in the classical regression model.

\begin{tabular}{|l|r|}
\hline$\hat{\boldsymbol{\alpha}}$ & \\
\hline$[1]$, & -37.54 \\
\hline$[2]$, & 5.42 \\
\hline
\end{tabular}

\begin{tabular}{|l|c|r|}
\hline$\hat{\boldsymbol{\beta}}$ & \multicolumn{1}{|c|}{$[, 1]$} & \multicolumn{1}{|c|}{$[, 2]$} \\
\hline$[1]$, & 0.82 & 0.044 \\
\hline$[2]$, & 0.52 & 0.86 \\
\hline
\end{tabular}

In the figure 1 we can see the measured (black) and estimated (bold red) values of blood flow through a bypass and blood pressure after removal of the cross clamp from the aorta. To compare the efficiency of the classical regression model and the "Crystal ball" model we show residuals in the following table $\left(\xi_{i}\right.$ and $\eta_{i}$ are measured values of blood flow and blood pressure after removal of the clamp from the aorta):

$\Delta v_{i, 1}=\xi_{i}-v_{i, 1}, \quad \Delta v_{i, 2}=\eta_{i}-v_{i, 2}, \quad i=1,2, \ldots, n$. 
Table 3. The residuals in the classical regression model.

\begin{tabular}{|l|r|r|}
\hline $\mathrm{i}$ & $\Delta v_{i, 1}$ & $\Delta v_{i, 2}$ \\
\hline 1 & 4.76 & -1.69 \\
\hline 2 & -29.08 & -3.36 \\
\hline 3 & -8.18 & -2.16 \\
\hline 4 & 17.85 & 0.94 \\
\hline 5 & 7.01 & 1.66 \\
\hline 6 & 2.62 & 5.95 \\
\hline 7 & -12.68 & 1.05 \\
\hline 8 & 16.82 & 1.14 \\
\hline 9 & 24.88 & -8.47 \\
\hline 10 & -2.24 & -4.56 \\
\hline 11 & -4.78 & 4.85 \\
\hline
\end{tabular}

\begin{tabular}{|l|r|r|}
\hline 12 & 5.34 & -1.51 \\
\hline 13 & -28.32 & -1.91 \\
\hline 14 & -15.59 & 1.16 \\
\hline 15 & -13.89 & 2.65 \\
\hline 16 & -6.47 & -8.32 \\
\hline 17 & 12.78 & 5.91 \\
\hline 18 & 5.57 & 4.33 \\
\hline 19 & 1.99 & 12.89 \\
\hline 20 & 1.22 & 3.92 \\
\hline 21 & 17.10 & 6.17 \\
\hline 22 & 17.85 & 1.47 \\
\hline 23 & 14.87 & -4.74 \\
\hline
\end{tabular}

\begin{tabular}{|l|r|r|}
\hline 24 & -0.94 & -2.20 \\
\hline 25 & -14.02 & 1.26 \\
\hline 26 & -11.09 & -7.33 \\
\hline 27 & -16.37 & -6.82 \\
\hline 28 & -3.12 & -0.63 \\
\hline 29 & 1.11 & -4.09 \\
\hline 30 & -1.75 & -6.29 \\
\hline 31 & 22.37 & 5.38 \\
\hline 32 & -4.50 & 1.13 \\
\hline 33 & -4.31 & -6.04 \\
\hline 34 & 4.89 & 4.08 \\
\hline 35 & -1.69 & 4.17 \\
\hline
\end{tabular}

\section{LIMA-LAD III}

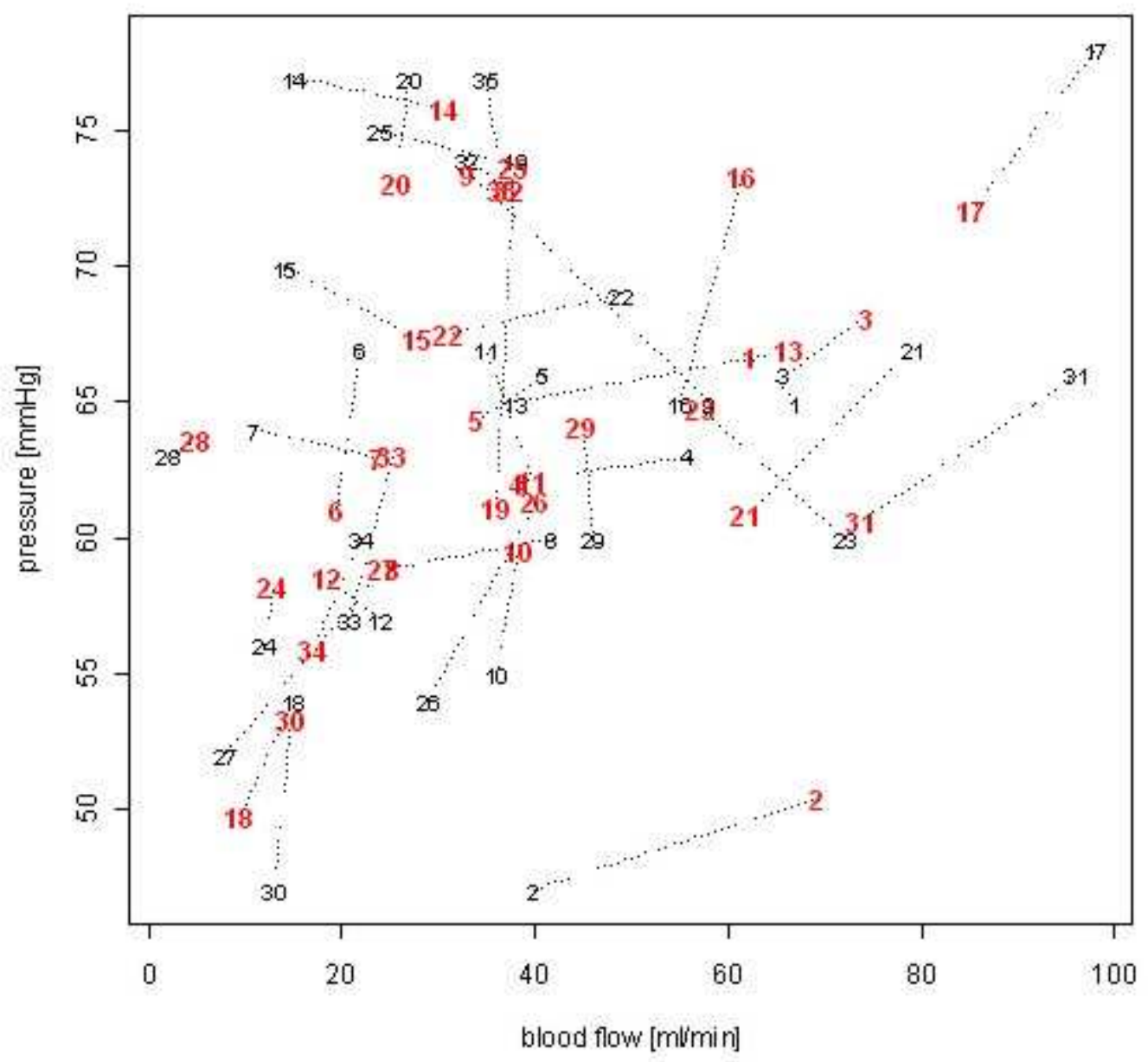

Fig. 1. Estimated and measured values of blood pressure and blood flow through a bypass after removal of the cross clamp from the aorta in the classical regression model. 


\subsection{The "Crystal ball" model}

We establish predicted values for patient number i as follows:

$$
\left(\begin{array}{c}
\hat{v}_{i, 1} \\
\hat{v}_{i, 2}
\end{array}\right)=\left(\begin{array}{l}
\hat{\beta}_{1} \\
\hat{\beta}_{2}
\end{array}\right)+\left(\begin{array}{ll}
\hat{\beta}_{3} & \hat{\beta}_{4} \\
\hat{\beta}_{5} & \hat{\beta}_{6}
\end{array}\right)\left(\begin{array}{l}
x_{i} \\
y_{i}
\end{array}\right),
$$

where $x_{i}$ and $y_{i}$ are measured values of blood flow and blood pressure at the time when the cross clamp is in the place on the aorta and $\hat{v}_{i, 1}$ and $\hat{v}_{i, 2}$ are predicted values of these parameters after removal of the cross clamp.

An algorithm of the calculation of estimations of parameters $\beta_{i}, \mathrm{i}=1,2, \ldots, 6$, which is iterative, is presented in the appendix A.1. Now we are testing the efficiency of the suggested algorithm on a data set which was used for the estimate of parameters $\beta_{i}$. We compare estimated values with directly measured values. The points for the initial iteration lie on a border of the data field ( $\mathrm{i}=14,17,18)$. The numbers mean order numbers of observations (patients).

Values of parameters $\beta_{i}$ obtained in six iterative steps for the points of initial iteration mentioned above and our data set are shown in the table 4.

Table 4. Estimated parameters in the "Crystal ball" model.

\begin{tabular}{|c|r|}
\hline$\hat{\beta}_{1}$ & -99.10 \\
\hline$\hat{\beta}_{2}$ & -11.96 \\
\hline$\hat{\beta}_{3}$ & 0.96 \\
\hline$\hat{\beta}_{4}$ & 1.34 \\
\hline$\hat{\beta}_{5}$ & 0.06 \\
\hline$\hat{\beta}_{6}$ & 1.12 \\
\hline
\end{tabular}

For our data set we obtain following variance matrix and estimation of $\sigma^{2}$ (the algorithm is presented in the appendix A.1): 


$$
\begin{aligned}
& \hat{\sigma}^{2}=46.52034, \\
& \operatorname{Var}\left(\begin{array}{l}
\hat{\beta}_{1} \\
\hat{\beta}_{2} \\
\hat{\beta}_{3} \\
\hat{\beta}_{4} \\
\hat{\beta}_{5} \\
\hat{\beta}_{6}
\end{array}\right)=\left(\begin{array}{rrrrrr}
539.914 & 225.030 & -0.840 & -7.528 & -0.350 & -3.137 \\
225.030 & 326.432 & -0.350 & -3.137 & -0.508 & -4.551 \\
-0.840 & -0.350 & 0.008 & 0.005 & 0.003 & 0.002 \\
-7.528 & -3.137 & 0.005 & 0.110 & 0.002 & 0.046 \\
-0.350 & -0.508 & 0.003 & 0.002 & 0.005 & 0.003 \\
-3.137 & -4.551 & 0.002 & 0.046 & 0.003 & 0.066
\end{array}\right) .
\end{aligned}
$$

For numerical expression of accuracy of estimates obtained in our model, we show values of the following residuals in the table 5:

$$
\Delta v_{i, 1}=\xi_{i}-\hat{v}_{i, 1}, \quad \Delta v_{i, 2}=\eta_{i}-\hat{v}_{i, 2}, \quad i=1,2, \ldots, n
$$

Table 5. The residuals in the "Crystal ball" model.

\begin{tabular}{|r|r|r|}
\hline \multicolumn{1}{|c|}{$\mathrm{i}$} & \multicolumn{1}{|c|}{$\Delta v_{i, 1}$} & \multicolumn{1}{c|}{$\Delta v_{i, 2}$} \\
\hline$[1]$ & 0.32 & -1.33 \\
\hline$[2]$ & -8.18 & 6.01 \\
\hline$[3]$ & -5.39 & 2.25 \\
\hline$[4]$ & 6.95 & -4.19 \\
\hline$[5]$ & 2.33 & -0.94 \\
\hline$[6]$ & 1.14 & 2.23 \\
\hline$[7]$ & -4.04 & 3.42 \\
\hline$[8]$ & 7.97 & -4.39 \\
\hline$[9]$ & 9.40 & -11.58 \\
\hline$[10]$ & 1.45 & -2.46 \\
\hline$[11]$ & -2.67 & 4.26 \\
\hline
\end{tabular}

\begin{tabular}{|r|r|r|}
\hline$[12]$ & 4.77 & -3.28 \\
\hline$[13]$ & -12.31 & 7.37 \\
\hline$[14]$ & -8.76 & 5.08 \\
\hline$[15]$ & -6.23 & 5.09 \\
\hline$[16]$ & -3.81 & -2.27 \\
\hline$[17]$ & -1.16 & 2.36 \\
\hline$[18]$ & 6.04 & -0.41 \\
\hline$[19]$ & -1.75 & 7.33 \\
\hline$[20]$ & -2.22 & 2.10 \\
\hline$[21]$ & 4.42 & 0.06 \\
\hline$[22]$ & 5.79 & -3.86 \\
\hline$[23]$ & 5.66 & -6.17 \\
\hline
\end{tabular}

\begin{tabular}{|r|r|r|}
\hline$[24]$ & 2.96 & -2.28 \\
\hline$[25]$ & -8.04 & 4.90 \\
\hline$[26]$ & -1.69 & -1.72 \\
\hline$[27]$ & -2.38 & -0.68 \\
\hline$[28]$ & 0.76 & -0.76 \\
\hline$[29]$ & 1.11 & -2.61 \\
\hline$[30]$ & 4.86 & -4.78 \\
\hline$[31]$ & 6.06 & -1.41 \\
\hline$[32]$ & -4.19 & 2.26 \\
\hline$[33]$ & 0.84 & -3.14 \\
\hline$[34]$ & 3.89 & 0.16 \\
\hline$[35]$ & -3.87 & 3.39 \\
\hline
\end{tabular}

In the figure 2, there are measured (black) and estimated (bold red) values of blood flow and blood pressure after removal of the cross clamp from the aorta. 


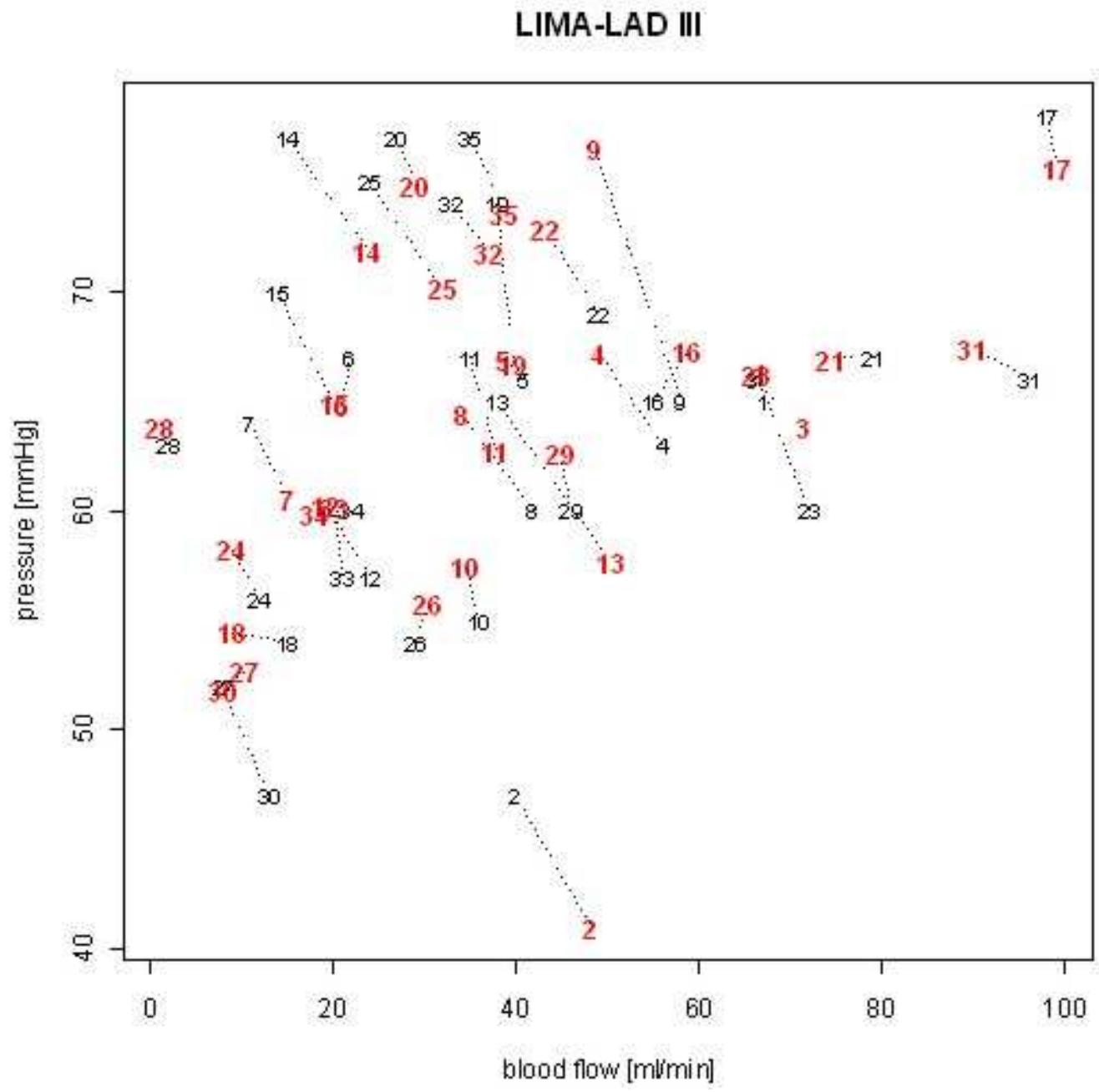

Fig. 2. Predicted and measured values of blood pressure and blood flow through a bypass after removal of the cross clamp in the "Crystal ball" model.

Already from the picture and also from the values of residuals we can see that the "Crystal ball" model, which contains more parameters and this fact leads to better approximation of the measured values by our estimates, describes reality better than the classical linear regression model.

\subsection{Location of outlier points in the "Crystal ball" model}

In this section, we try to locate outlier observations in the "Crystal ball" model. Thereafter, we will exclude the outliers from the data set used in calculation of parameters and study changes in the model.

We find the indexes for which the expressions (35) are greater than or equal to 1.96 (or approximately 2). The points of these indexes are our suspect outliers.

For our data, set we have found as outliers the points with indexes $2,9,13,14,25$, as you can see in the table 6. 
Table 6. Suspect outliers in the "Crystal ball" model.

\begin{tabular}{|c|c|c|}
\hline i & $\left\{\mathrm{v}_{1}\right\} / \sqrt{\left(\hat{\operatorname{Var}}\left(\mathrm{v}_{1}\right)\right\}}$ & $\left\{\mathrm{v}_{2}\right\} / \sqrt{\left.\hat{\operatorname{V}} \operatorname{Var}\left(\mathrm{v}_{2}\right)\right\}_{0}}$ \\
\hline 1 & -0.0620 & 0.0795 \\
\hline 1 & 0.1952 & -0.2574 \\
\hline 2 & 2.4664 & -2.4933 \\
\hline 2 & 0.9999 & 1.4239 \\
\hline 3 & 1.3696 & -1.3573 \\
\hline 3 & 0.9032 & 0.4407 \\
\hline 4 & -1.6880 & 1.6916 \\
\hline 4 & -0.8654 & -0.7923 \\
\hline 5 & -0.5717 & 0.5661 \\
\hline 5 & -0.3838 & -0.1771 \\
\hline 6 & -0.3219 & 0.2789 \\
\hline 6 & -0.7385 & 0.4257 \\
\hline 7 & 0.9763 & -0.9934 \\
\hline 7 & 0.3050 & 0.6549 \\
\hline 8 & -1.9367 & 1.9347 \\
\hline 8 & -1.0731 & -0.8284 \\
\hline 9 & -2.2851 & 2.3823 \\
\hline 9 & 0.0311 & -2.2822 \\
\hline 10 & -0.3321 & 0.3563 \\
\hline 10 & 0.1412 & -0.4691 \\
\hline 11 & 0.6068 & -0.6475 \\
\hline 11 & -0.2048 & 0.8037 \\
\hline 12 & -1.1603 & 1.1688 \\
\hline 12 & -0.5146 & -0.6253 \\
\hline 13 & 3.0490 & -3.0550 \\
\hline 13 & 1.5730 & 1.4212 \\
\hline 14 & 2.2011 & -2.2022 \\
\hline 14 & 1.1691 & 0.9922 \\
\hline 15 & 1.5050 & -1.5281 \\
\hline 15 & 0.5091 & 0.9703 \\
\hline 16 & 1.0020 & -0.9355 \\
\hline 16 & 1.4110 & -0.4322 \\
\hline 17 & 0.2890 & -0.3170 \\
\hline 17 & -0.2156 & 0.5015 \\
\hline 18 & -1.5652 & 1.5185 \\
\hline
\end{tabular}

\begin{tabular}{|r|r|r|}
\hline $\mathrm{i}$ & $\left\{\mathrm{v}_{1}\right\} / \sqrt{\left.\hat{\operatorname{Var}}\left(\mathrm{v}_{1}\right)\right\}}$ & $\left\{\mathrm{v}_{2}\right\} / \sqrt{\left(\hat{\operatorname{Var}}\left(\mathrm{v}_{2}\right)\right\}}$ \\
\hline 18 & -1.4524 & -0.0809 \\
\hline 19 & 0.3311 & -0.4250 \\
\hline 19 & -1.0514 & 1.3836 \\
\hline 20 & 0.5530 & -0.5654 \\
\hline 20 & 0.1288 & 0.4152 \\
\hline 21 & -1.1524 & 1.1128 \\
\hline 21 & -1.1412 & 0.0126 \\
\hline 22 & -1.4256 & 1.4346 \\
\hline 22 & -0.6574 & -0.7431 \\
\hline 23 & -1.3583 & 1.4030 \\
\hline 23 & -0.1483 & -1.1888 \\
\hline 24 & -0.7258 & 0.7348 \\
\hline 24 & -0.2723 & -0.4410 \\
\hline 25 & $\mathbf{1 . 9 7 6 8}$ & $-\mathbf{1 . 9 8 1 4}$ \\
\hline 25 & 1.0032 & 0.9382 \\
\hline 26 & 0.4584 & -0.4183 \\
\hline 26 & 0.7779 & -0.3309 \\
\hline 27 & 0.6324 & -0.6015 \\
\hline 27 & 0.7532 & -0.1346 \\
\hline 28 & -0.1868 & 0.1920 \\
\hline 28 & -0.0345 & -0.1493 \\
\hline 29 & -0.2397 & 0.2690 \\
\hline 29 & 0.2549 & -0.4925 \\
\hline 30 & -1.2062 & 1.2371 \\
\hline 30 & -0.2399 & -0.9468 \\
\hline 31 & -1.6281 & 1.5954 \\
\hline 31 & -1.3074 & -0.2888 \\
\hline 32 & 1.0383 & -1.0361 \\
\hline 32 & 0.5853 & 0.4341 \\
\hline 33 & -0.1648 & 0.2045 \\
\hline 33 & 0.4332 & -0.5981 \\
\hline 34 & -0.9889 & 0.9534 \\
\hline 34 & -0.9984 & 0.0301 \\
\hline 35 & 0.9476 & -0.9653 \\
\hline 35 & 0.2739 & 0.6579 \\
\hline
\end{tabular}

After exclusion of our suspect points from calculation of parameters in the "Crystal ball" model, we obtain the results shown in the table 7.

When we compare values of calculated parameters in the case of an entire data set and a data set with exclusion of suspect outliers, we can see that the blood flow after removal of the cross clamp depends more on the blood flow at the time when the cross clamp is applied to the aorta and much less on the blood pressure at the time when the cross clamp is in place on the aorta. Moreover, we can claim that the differences in dependence on blood flow (less in 
the entire data set) and blood pressure (higher in the entire data) get bigger in a case of blood pressure after removal of the cross clamp.

Table 7. Estimated parameters after exclusion of the suspect outliers.

\begin{tabular}{|c|r|}
\hline$\hat{\beta}_{1}$ & -45.67 \\
\hline$\hat{\beta}_{2}$ & -19.17 \\
\hline$\hat{\beta}_{3}$ & 1.04 \\
\hline$\hat{\beta}_{4}$ & 0.50 \\
\hline$\hat{\beta}_{5}$ & 0.05 \\
\hline$\hat{\beta}_{6}$ & 1.24 \\
\hline
\end{tabular}

\section{LIMA-LAD III}

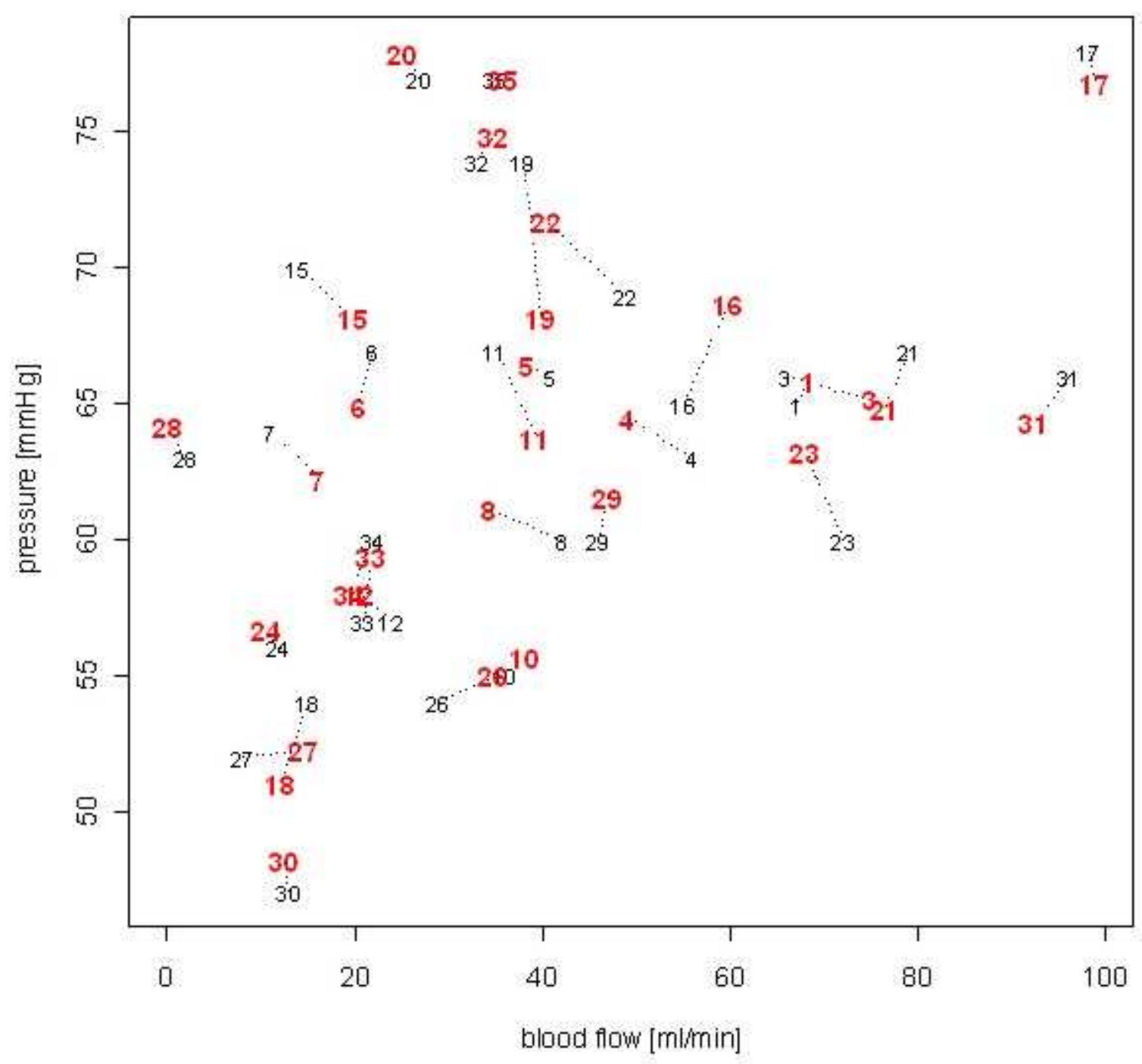


Fig. 3. Predicted and measured values of blood pressure and blood flow through a bypass after removal of the cross clamp from the aorta after exclusion of suspect outliers.

Already in the figure 3, where estimates of blood flow are marked in bold red and blood pressure and in black, we can see that the residuals got lower after the exclusion of suspect outliers. Also, the table of residuals confirms this statement.

Table 8. Residuals after exclusion of the suspect outliers.

\begin{tabular}{|l|r|r|}
\hline $\mathrm{i}$ & \multicolumn{1}{|c|}{$\Delta v_{i, 1}$} & \multicolumn{1}{c|}{$\Delta v_{i, 2}$} \\
\hline 1 & -1.40 & -0.86 \\
\hline 3 & -8.74 & 0.83 \\
\hline 4 & 6.91 & -1.50 \\
\hline 5 & 2.66 & -0.45 \\
\hline 6 & 1.55 & 2.04 \\
\hline 7 & -5.27 & 1.73 \\
\hline 8 & 7.68 & -1.15 \\
\hline 10 & -2.13 & -0.67 \\
\hline 11 & -4.31 & 3.19 \\
\hline 12 & 3.48 & -0.98 \\
\hline
\end{tabular}

\begin{tabular}{|r|r|r|}
\hline $\mathbf{i}$ & \multicolumn{1}{|c|}{$\Delta v_{i, 1}$} & \multicolumn{1}{c|}{$\Delta v_{i, 2}$} \\
\hline 15 & -5.96 & 1.80 \\
\hline 16 & -4.77 & -3.69 \\
\hline 17 & -0.88 & 1.15 \\
\hline 18 & 2.84 & 2.99 \\
\hline 19 & -1.79 & 5.79 \\
\hline 20 & 2.00 & -0.97 \\
\hline 21 & 2.60 & 2.13 \\
\hline 22 & 8.55 & -2.76 \\
\hline 23 & 4.09 & -3.29 \\
\hline 24 & 1.35 & -0.64 \\
\hline
\end{tabular}

\begin{tabular}{|r|r|r|}
\hline $\mathbf{i}$ & \multicolumn{1}{|c|}{$\Delta v_{i, 1}$} & \multicolumn{1}{c|}{$\Delta v_{i, 2}$} \\
\hline 26 & -5.90 & -0.99 \\
\hline 27 & -6.69 & -0.22 \\
\hline 28 & 1.94 & -1.19 \\
\hline 29 & -0.87 & -1.60 \\
\hline 30 & 0.54 & -1.13 \\
\hline 31 & 3.57 & 1.65 \\
\hline 32 & -1.90 & -0.86 \\
\hline 33 & -0.70 & -2.34 \\
\hline 34 & 2.42 & 1.95 \\
\hline 35 & -0.86 & 0.04 \\
\hline
\end{tabular}

\subsection{Location of leverage points in the "Crystal ball" model}

In this section, we find points which most affect the values of estimated blood flow and blood pressure in the "Crystal ball" model (section 1.2, equations (2), (3)).

We want to find the maximum of absolute values of derivation of the terms (38) and (39), i.e. maximum of:

$$
\left|\frac{\partial\left\{\boldsymbol{\Pi}_{\mathbf{1}} \mathbf{Z}\right\}_{i}}{\partial\{\mathbf{Z}\}_{j}}\right|=\left|\left\{\boldsymbol{\Pi}_{\mathbf{1}}\right\}_{i, j}\right| \quad \text { and } \quad\left|\frac{\partial \hat{\beta}}{\partial\{\mathbf{Z}\}_{j}}\right|=-\left\{\boldsymbol{\Pi}_{\mathbf{2}}\right\}_{i, j} \mid \text {. }
$$

For our data set without the exclusion of suspected outliers, we obtain by calculation as a leverage point for estimates of increments $\delta \boldsymbol{\mu}, \boldsymbol{\delta} \boldsymbol{v}$ and also for parameters $\beta$ the point with index 2 . After the exclusion of suspected outliers, we obtain as leverage point for estimates of increments $\delta \boldsymbol{\mu}$ and $\boldsymbol{\delta} \boldsymbol{v}$ the point with index 17 and for estimate of increments of parameters $\beta$ the point with index 30 . 


\section{Conclusions}

If we use the classical linear model for estimate of blood pressure and blood flow after removal of the cross clamp from the aorta during CABG surgery, we obtain less accurate estimates than when we use the "Crystal ball" model. Moreover, when we exclude suspected outliers our estimates are even more accurate. This statement leads to the hypothesis that patients are divided into several groups and with our algorithm we can calculate certain values of parameters for one group and generally different values for other groups. However, inclusion of patients in the wrong group may be manifested as outliers. To confirm this hypothesis we must complete a deeper analysis of a larger group of patients and include in the surveyed parameters those which can affect blood flow through a bypass before and after removal of the cross clamp from the aorta in the way that they define the above mentioned groups. We can use for example the percentage of stenosis in the target coronary bed, the ejection fraction, FFT ratio, the type of bypass used (LIMA, RIMA, SVG), time of dilatation, the bypass as pedicle/scelet and others [2], [3], [12].

In this orientation period of research a very simplified structure of covariance matrix was used. In further research the covariation matrix, which respects differences in dispersions of the measure of blood flow and the measure of blood pressure must be studied.

The numerical results obtained by suggested method the "Crystal ball" indicate real possibility to predict values of blood flow and blood pressure after removal of the cross clamp from the aorta. However, many calculations on larger data sets must be done before release of this method.

\section{Appendix The "Crystal ball" model}

The model of the relationship between blood flow and pressure at the time when the clamp is applied to the aorta (LIMA-LAD I) and at the time when the clamp is removed (LIMA-LAD III) we assume in this form (for notation see section 1.1):

$$
E(\zeta)\left[\square \left[\square_{1,1}, \square_{1,2}, \ldots, \square_{n, 1}, \square_{n, 2}, \square_{1,1}, \square_{1,2}, \ldots, \square_{n, 1}, \square_{n, 2}\left[, \operatorname{Var}(\zeta) \square \square^{2}\left(\begin{array}{cc}
2 n, \boldsymbol{Q} & \mathbf{I} \\
& 2 n, 2 n
\end{array}\right),\right.\right.\right.
$$

where $E(\zeta)$ satisfies the conditions:

$$
\left(\begin{array}{l}
E\left(\xi_{i}\right) \\
E\left(\eta_{i}\right)
\end{array}\right)=\left(\begin{array}{l}
\beta_{1} \\
\beta_{2}
\end{array}\right)+\left(\begin{array}{ll}
\beta_{3} & \beta_{4} \\
\beta_{5} & \beta_{6}
\end{array}\right)\left(\begin{array}{l}
E\left(x_{i}\right) \\
E\left(y_{i}\right)
\end{array}\right), \quad i=1,2, \ldots, n
$$

Note: $E(\zeta)$ and $\operatorname{Var}(\zeta)$ denote the mean value and the variance of the random vector $\zeta$. 


\section{A.1 Estimate of parameters of the model}

The task is to estimate values of model parameters, i.e. $\mu_{1,1}, \mu_{1,2}, \ldots, \mu_{n, 1}, \mu_{n, 2}, v_{1,1}, v_{1,2}, \ldots, v_{n, 1}, v$ ${ }_{n, 2}$ and namely $\beta_{1}, \ldots, \beta_{6}$, in terms of our measured data. To solve this problem we use linearization of the model. The model is not linear because there are products of parameters $\boldsymbol{\beta}$ and $\boldsymbol{\mu}$. After linearization we use estimate algorithms from the model of a direct incomplete measurement of a vector parameter with a system of constraints from the fourth section of the book Statistika a metrologie [14] and from the book Statistical models with Linear Structures [15]. A necessary condition for estimation of the parameters is the number of observations plus the number of constraints has to be greater than a number of the parameters, in our case: $4 n+2 n>4 n+6$, because we measure four parameters of each patient.

After linearization we get the model of a direct incomplete measurement of a vector parameter:

$$
\left.\zeta \square\left(\begin{array}{c}
\square_{1,1}^{(0)} \\
\sqcup_{1,2}^{(0)} \\
\vdots \\
\square_{n, 1}^{(0)} \\
\square_{n, 2}^{(0)}
\end{array}\right) \sim\left(\begin{array}{c}
\square_{140} \\
\square_{1,1} \\
\vdots \\
\square_{n, 1} \\
\square_{n, 2}
\end{array}\right) ; \square^{2} \mathbf{I}\right)
$$

with constraints

$$
\mathbf{b} \square \mathbf{B}_{\mathbf{1}}\left(\begin{array}{c}
\square_{1,1} \\
\square_{1,2} \\
\vdots \\
\square_{n, 1} \\
\square_{n, 2}
\end{array}\right) \square \mathbf{B}_{2}\left(\begin{array}{c}
\square_{1} \\
\vdots \\
\square_{6}
\end{array}\right) \square 0
$$


where

$$
\mathbf{b}=\left(\begin{array}{c}
-v_{1,}^{(0)}+\beta_{1}^{(0)}+\beta_{3}^{(0)} \mu_{1,}^{(0)}+\beta_{4}^{(0)} \mu_{1,2}^{(0)} \\
\vdots \\
-v_{i, 1}^{(0)}+\beta_{1}^{(0)}+\beta_{3}^{(0)} \mu_{i, 1}^{(0)}+\beta_{4}^{(0)} \mu_{i, 2}^{(0)} \\
-v_{i, 2}^{(0)}+\beta_{2}^{(0)}+\beta_{4}^{(0)} \mu_{i,}^{(0)}+\beta_{6}^{(0)} \mu_{1,2}^{(0)} \\
\vdots \\
-v_{i, 2}^{(0)}+\beta_{2}^{(0)}+\beta_{4}^{(0)} \mu_{i, 1}^{(0)}+\beta_{6}^{(0)} \mu_{1,2}^{(0)}
\end{array}\right) .
$$

The matrix $\mathbf{B}_{1}$ of the type $2 \mathrm{nx} 4 \mathrm{n}$ is (a vertical line separates first $2 \mathrm{n}$ columns):

$$
\left(\begin{array}{ccccccccc:ccccccccc}
\beta_{3}^{(0)} & \beta_{4}^{(0)} & 0 & 0 & 0 & \cdots & 0 & 0 & 0 & -1 & 0 & 0 & 0 & 0 & \cdots & 0 & 0 & 0 \\
\beta_{5}^{(0)} & \beta_{6}^{(0)} & 0 & 0 & 0 & \cdots & 0 & 0 & 0 & 0 & -1 & 0 & 0 & 0 & \cdots & 0 & 0 & 0 \\
0 & 0 & \beta_{3}^{(0)} & \beta_{4}^{(0)} & 0 & \cdots & 0 & 0 & 0 & 0 & 0 & -1 & 0 & 0 & \cdots & 0 & 0 & 0 \\
0 & 0 & \beta_{5}^{(0)} & \beta_{6}^{(0)} & 0 & \cdots & 0 & 0 & 0 & 0 & 0 & 0 & -1 & 0 & \cdots & 0 & 0 & 0 \\
\cdots & \cdots & \cdots & \cdots & \cdots & \cdots & \cdots & \cdots & \cdots & \cdots & \cdots & \cdots & \cdots & \cdots & \cdots & \cdots & \cdots & \cdots \\
0 & 0 & 0 & 0 & 0 & \cdots & 0 & \beta_{3}^{(0)} & \beta_{4}^{(0)} & 0 & 0 & 0 & 0 & 0 & \cdots & 0 & -1 & 0 \\
0 & 0 & 0 & 0 & 0 & \cdots & 0 & \beta_{s}^{(0)} & \beta_{6}^{(0)} & 0 & 0 & 0 & 0 & 0 & \cdots & 0 & 0 & -1
\end{array}\right) .
$$

The matrix $\mathbf{B}_{\mathbf{2}}$ of the type $2 \mathrm{nx} 6$ is:

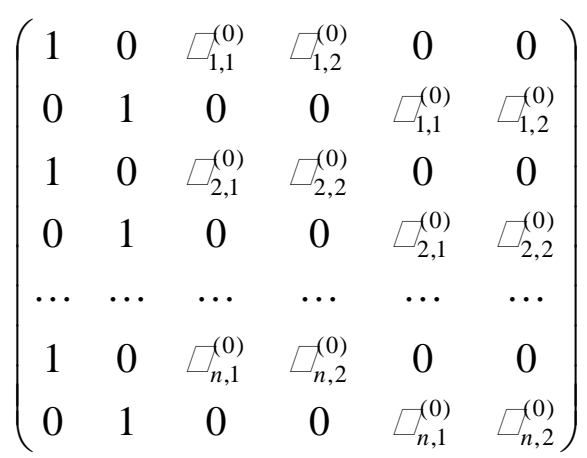


Estimations of parameters $\delta \mu_{i, j}, \delta v_{i, j}, i=1,2, \ldots, n, j=1,2$ and $\delta \beta_{i}, i=1,2, \ldots, 6$ are obtained by minimization of function:

$$
\square \square \rrbracket_{1,1}, \cdots, \square_{n, 2} \square\left(\zeta \square\left(\begin{array}{c}
\square_{1,1}^{(0)} \\
\vdots \\
\square_{n, 2}^{(0)}
\end{array}\right) \square\left(\begin{array}{c}
\square \\
\square \\
\square_{n, 2}
\end{array}\right)\right)\left(\zeta \square\left(\begin{array}{c}
\square_{1,1}^{(0)} \\
\vdots \\
\square_{n, 2}^{(0)}
\end{array}\right) \square\left(\begin{array}{c}
\square_{1,1} \\
\vdots \\
\square \\
\square, 2
\end{array}\right)\right)
$$

with satisfaction of the constraints (11).

After tedious calculation we get the result in the form:

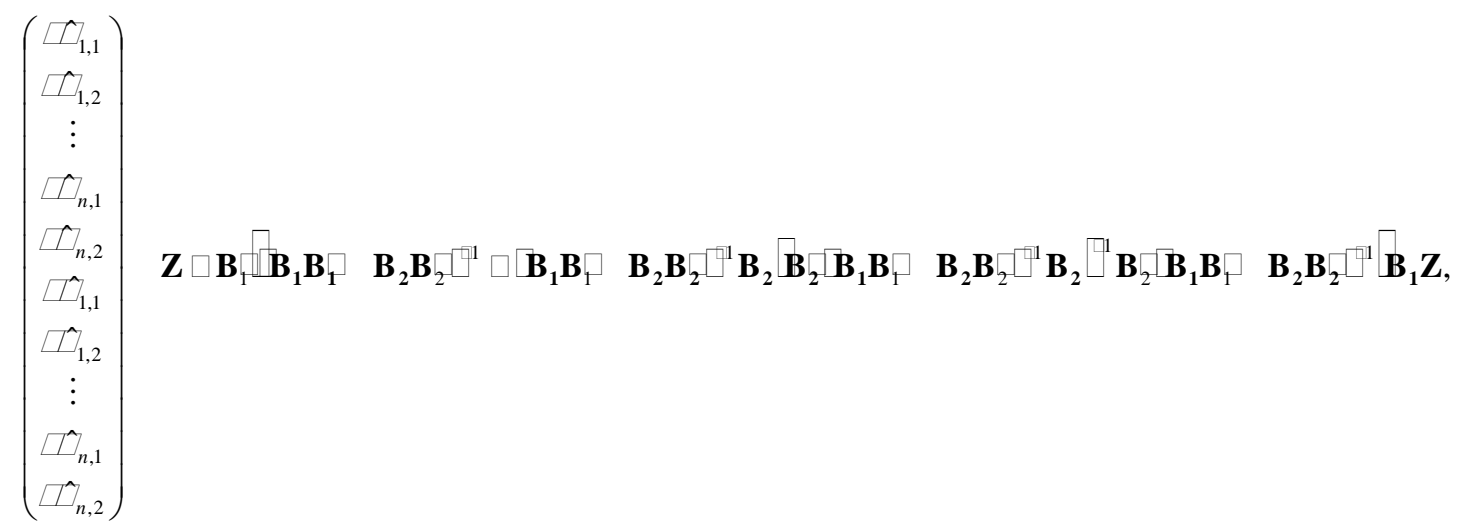

$\left(\hat{\delta} \hat{\beta}_{1}, \delta \hat{\beta}_{2}, \delta \hat{\beta}_{3}, \delta \hat{\beta}_{4}, \delta \hat{\beta}_{5}, \delta \hat{\beta}_{6}\right)^{t}=-\left[\mathbf{B}_{2}^{t}\left(\mathbf{B}_{1} \mathbf{B}_{1}^{t}+\mathbf{B}_{2} \mathbf{B}_{2}^{t}\right)^{-1} \mathbf{B}_{2}\right]^{-1} \mathbf{B}_{2}^{t}\left(\mathbf{B}_{\mathbf{1}} \mathbf{B}_{\mathbf{1}}^{t}+\mathbf{B}_{2} \mathbf{B}_{2}^{t}\right)^{-1} \mathbf{B}_{1} \mathbf{Z}$,

where

$$
\mathbf{Z} \square \square x_{1} \square \square_{1,1}^{(0)}, y_{1} \square \square_{1,2}^{(0)}, \ldots, x_{n} \square \square_{n, 1}^{(0)}, y_{n} \square \square_{n, 2}^{(0)}, \square_{1} \square \square_{1,1}^{(0)}, \square_{1} \square \square_{1,2}^{(0)}, \ldots, \square_{n} \square \square_{n, 1}^{(0)}, \square_{n} \square \nearrow_{n, 2}^{(0)}\lfloor.
$$

This result is considered as the result of the first iteration step, i.e.:

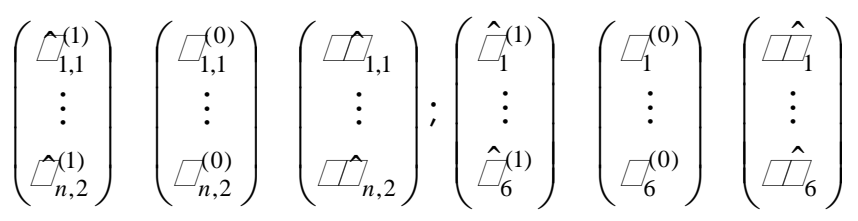


Following vectors are used in the next iteration step

$$
\left(\begin{array}{c}
\hat{\mu}_{1,1}^{(1)} \\
\vdots \\
\hat{v}_{n, 2, \text { korig. }}^{(1)}
\end{array}\right),\left(\begin{array}{c}
\hat{\beta}_{1}^{(1)} \\
\vdots \\
\hat{\beta}_{6}^{(1)}
\end{array}\right) \text { instead of vectors }\left(\begin{array}{c}
\mu_{1,1}^{(0)} \\
\vdots \\
\nu_{n, 2}^{(0)}
\end{array}\right),\left(\begin{array}{c}
\beta_{1}^{(0)} \\
\vdots \\
\beta_{6}^{(0)}
\end{array}\right) \text {. }
$$

The resulting estimate can be written as follows:

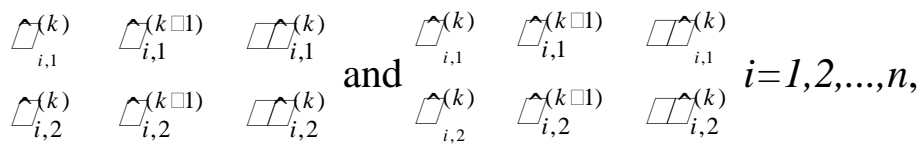

$$
\begin{aligned}
& \hat{\zeta}_{i}^{(k)} \square \hat{\zeta}_{i}^{(k \square 1)} \square \hat{U}_{i}^{(k)} i=1,2, \ldots, 6,
\end{aligned}
$$

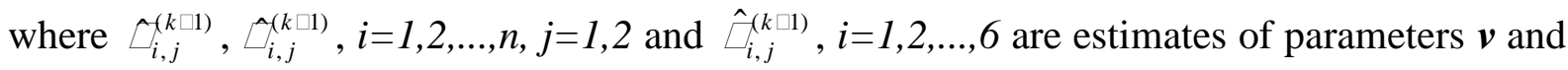
$\boldsymbol{\beta}$ from the previous iteration.

The vector $\mathbf{Z}^{(\mathbf{k})}$ in the k-th iteration step is:

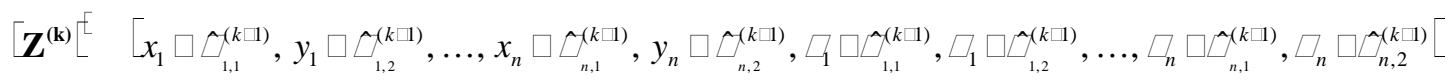

In the iterative process we correct the estimates of the parameters $v$ with these relationships:

$$
\begin{aligned}
& \hat{v}_{i, 1 \text {,orig }}^{(k)}=\hat{\beta}_{1}^{(k)}+\hat{\beta}_{3}^{(k)} \hat{\mu}_{i, 1}^{(k)}+\hat{\beta}_{4}^{(k)} \hat{\mu}_{i, 2}^{(k)} \\
& \hat{v}_{i, 2 \text {, forig. }}^{(k)}=\hat{\beta}_{2}^{(k)}+\hat{\beta}_{s}^{(k)} \hat{\mu}_{i, 1}^{(k)}+\hat{\beta}_{6}^{(k)} \hat{\mu}_{i, 2}^{(k)}
\end{aligned}
$$

For the initial (zero) iteration we choose

$$
\sqcup_{i, 1}^{(0)} \square x_{i}, \quad \square_{i, 2}^{(0)} \square y_{i} \quad \text { for } i=1,2, \ldots, n
$$

and parameters $v_{i, j}{ }^{(0)}, i=1,2, \ldots, n, j=1,2$ and $\beta_{i}{ }^{(0)}, i=1,2, \ldots, 6$ we calculate from equations for three points (in the xy plane), which lay at borders of points field, i.e. for certain coordinates: 


$$
\left(\begin{array}{l}
x_{i 1} \\
y_{i 1}
\end{array}\right),\left(\begin{array}{l}
x_{i 2} \\
y_{i 2}
\end{array}\right),\left(\begin{array}{l}
x_{i 3} \\
y_{i 3}
\end{array}\right) \text { and corresponding points }\left(\begin{array}{l}
\xi_{i 1} \\
\eta_{i 1}
\end{array}\right),\left(\begin{array}{l}
\xi_{i 2} \\
\eta_{i 2}
\end{array}\right),\left(\begin{array}{c}
\xi_{i 3} \\
\eta_{i 3}
\end{array}\right) \text {. }
$$

For these points we solve the system of equations:

$$
\left(\begin{array}{cccccc}
1 & 0 & x_{i 1} & y_{i 1} & 0 & 0 \\
0 & 1 & 0 & 0 & x_{i 1} & y_{i 1} \\
1 & 0 & x_{i 2} & y_{i 2} & 0 & 0 \\
0 & 1 & 0 & 0 & x_{i 2} & y_{i 2} \\
1 & 0 & x_{i 3} & y_{i 3} & 0 & 0 \\
0 & 1 & 0 & 0 & x_{i 3} & y_{i 3}
\end{array}\right)\left(\begin{array}{l}
\beta_{1}^{(0)} \\
\beta_{2}^{(0)} \\
\beta_{3}^{(0)} \\
\beta_{4}^{(0)} \\
\beta_{s}^{(0)} \\
\beta_{6}^{(0)}
\end{array}\right)=\left(\begin{array}{c}
\xi_{i 1} \\
\eta_{i 1} \\
\xi_{i 2} \\
\eta_{i 2} \\
\xi_{i 3} \\
\eta_{i 3}
\end{array}\right) .
$$

Parameters $\beta_{i}^{(0)}, i=1,2, \ldots, 6$ are the solution of this system and they are used for the calculation of the initial iteration of parameters $v_{i, j}^{(0)}, i=1,2, \ldots, n, j=1,2$ in following equations:

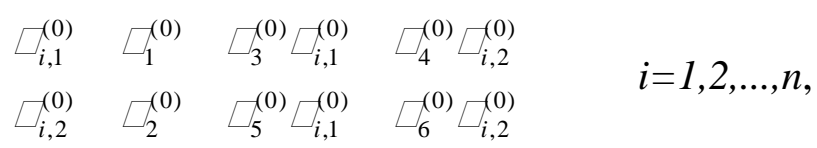

where parameters $\mu_{i, j}{ }^{(0)}, i=1,2, \ldots, n, j=1,2$ are set up in this way:

$\square_{i, 1}^{(0)} \square x_{i} \quad$ and $\quad \square_{i, 2}^{(0)} \square y_{i}$.

The iterative calculation is finished when estimates of the increments $\delta \mu_{i, j}, \delta v_{i, j}, i=1,2, \ldots, n$, $j=1,2$ and $\delta \beta_{i}, i=1,2, \ldots, 6$ can be omitted, i.e. on the following condition:

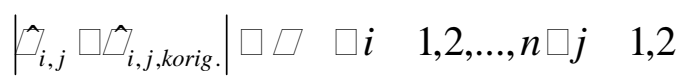

where $\varepsilon$ is a preset constant.

Estimates of parameters $\beta_{i}$ obtained in six iterative steps $(\varepsilon=0,1)$ and our data set are shown in the table 4 .

An estimator of a covariance matrix for these parameters is calculated by following relationship: 


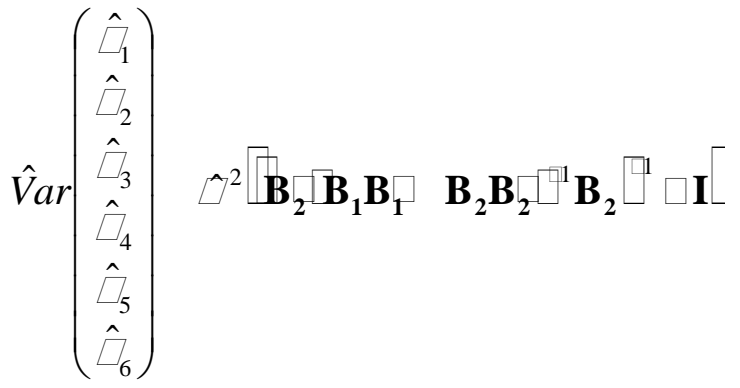

where

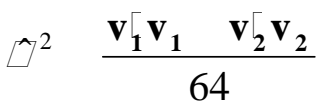

whereas

$64=$ number of measurements(140)+number of constraints(70)-number of parameters $(140+6)$

and

$\left(\begin{array}{c}\mathbf{v}_{1} \\ \mathbf{v}_{2}\end{array}\right)\left(\begin{array}{c}x_{1} \square \iota_{1,1} \\ \vdots \\ y_{n} \square \iota_{n, 2} \\ \hdashline \vdots \ldots \hat{\iota}_{1,1} \\ \vdots \\ \square, \hat{\iota}_{n, 2}\end{array}\right)$

For our data set we obtain following numerical results:

$$
\hat{\sigma}^{2}=46.52034,
$$

$$
\hat{\operatorname{Var}}\left(\begin{array}{l}
\hat{\beta}_{1} \\
\hat{\beta}_{2} \\
\hat{\beta}_{3} \\
\hat{\beta}_{4} \\
\hat{\beta}_{5} \\
\hat{\beta}_{6}
\end{array}\right)=\left(\begin{array}{rrrrrr}
539.914 & 225.030 & -0.840 & -7.528 & -0.350 & -3.137 \\
225.030 & 326.432 & -0.350 & -3.137 & -0.508 & -4.551 \\
-0.840 & -0.350 & 0.008 & 0.005 & 0.003 & 0.002 \\
-7.528 & -3.137 & 0.005 & 0.110 & 0.002 & 0.046 \\
-0.350 & -0.508 & 0.003 & 0.002 & 0.005 & 0.003 \\
-3.137 & -4.551 & 0.002 & 0.046 & 0.003 & 0.066
\end{array}\right) .
$$




\section{A.2 Location of outlier points in the "Crystal ball" model}

When the iteration is stopped (k-th step), for our vector of residuals $\mathbf{Z}^{(\mathbf{k})}$ from the equation (21), the following relationship is certainly valid:

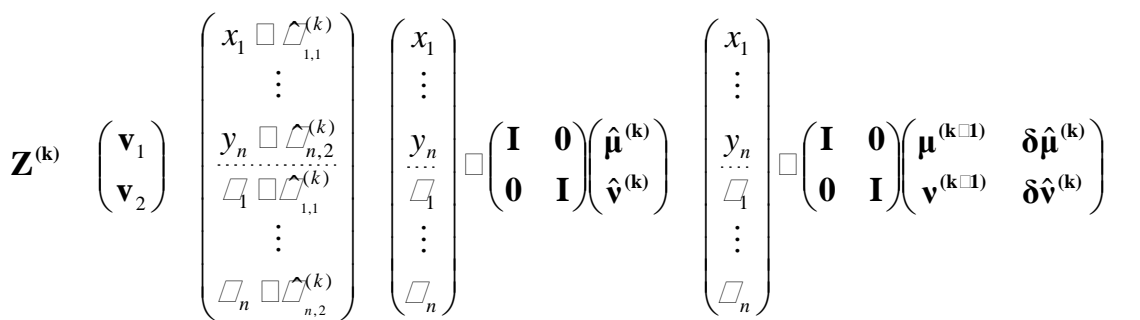

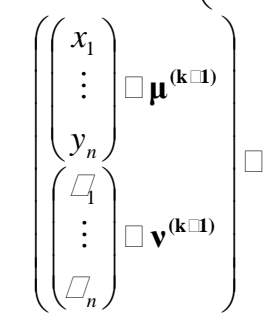

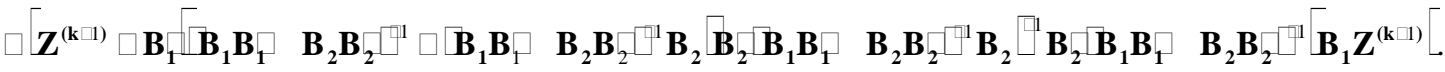

When we substitute the vector $\mathbf{Z}$ from the last iteration into the last row of expression we can rewrite the relationship in the following form:

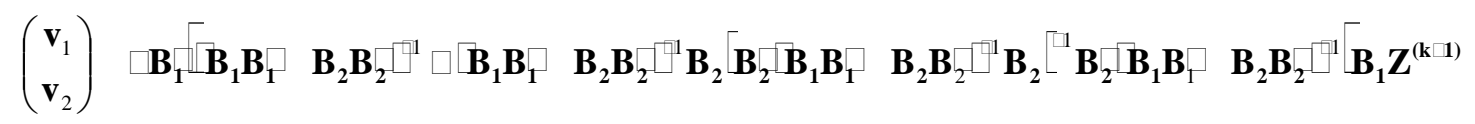

Thereafter, when we denote

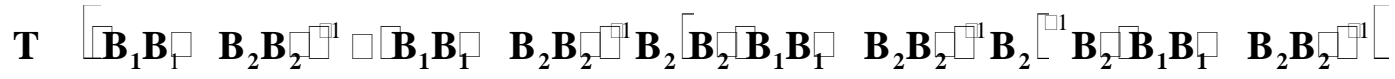

we can write

$\operatorname{Var}\left(\begin{array}{c}\mathbf{v}_{1} \\ \mathbf{v}_{2}\end{array}\right) \square \square^{2} \mathbf{B}_{\mathbf{1}} \mathbf{T B}_{\mathbf{1}} \mathbf{B}_{\mathbf{1}} \mathbf{T} B_{\mathbf{1}} \square\left(\begin{array}{cc}\operatorname{Var}\left(\mathbf{v}_{\mathbf{1}}\right) & \operatorname{cov}\left(\mathbf{v}_{\mathbf{1}}, \mathbf{v}_{\mathbf{2}}\right) \\ \operatorname{cov}\left(\mathbf{v}_{\mathbf{2}}, \mathbf{v}_{\mathbf{1}}\right) & \operatorname{Var}\left(\mathbf{v}_{\mathbf{2}}\right)\end{array}\right) \square \square^{2}\left(\begin{array}{cc}\mathbf{Q}_{11} & \mathbf{Q}_{\mathbf{1 2}} \\ \mathbf{Q}_{\mathbf{2 1}} & \mathbf{Q}_{\mathbf{2 2}}\end{array}\right)$

For every $i=1,2, \ldots, 2 n$ we calculate these expressions:

$$
\begin{aligned}
& \left|\nabla \mathbf{v}_{\mathbf{1}}{ }_{i}\right| / \sqrt{\hat{\operatorname{Var}}\left(\mathbf{v}_{\mathbf{1}}\right)_{i i}} \square\left|\overrightarrow{\mathbf{v}_{\mathbf{1}}} \square_{i}\right| / \sqrt{\hat{\iota}^{2}\left[\mathbf{Q}_{\mathbf{1 1}}\right.} \\
& \left|\nabla \mathbf{v}_{\mathbf{2}}{ }_{i}\right| / \sqrt{\hat{\operatorname{Var}}\left(\mathbf{v}_{\mathbf{2}}\right)_{i i}} \square\left|\nabla \mathbf{v}_{\mathbf{2}}{ }_{i}\right| / \sqrt{\hat{\iota}^{2} \mathbf{Q}_{22} \square_{i i}}
\end{aligned}
$$




\section{A.3 Location of leverage points in the "Crystal ball" model}

When the iteration process is stopped (k-th step) we use equations for increments

$$
\begin{aligned}
& \left(\begin{array}{c}
\square_{1,1} \\
\square_{1,2} \\
\vdots \\
\square_{n, 1} \\
\square_{n, 2} \\
\square \square_{1,1}
\end{array} \square \mathbf{Z}^{(k)} \square \mathbf{B}_{1}^{\square} \mathbf{B}_{1} \mathbf{B}_{1} \square \mathbf{B}_{2} \mathbf{B}_{2}^{\square} \square \mathbf{B}_{1} \mathbf{B}_{1} \square \mathbf{B}_{2} \mathbf{B}_{2}^{\square} \mathbf{B}_{2} \mathbf{B}_{2} \mathbf{B}_{1} \mathbf{B}_{1} \square \mathbf{B}_{2} \mathbf{B}_{2}^{\square} \mathbf{B}_{2}^{\square} \square \mathbf{B}_{2} \mathbf{B}_{1} \mathbf{B}_{1} \square \mathbf{B}_{2} \mathbf{B}_{2}^{\square} \mathbf{B}_{1} \mathbf{Z}^{(k)}\right.
\end{aligned}
$$

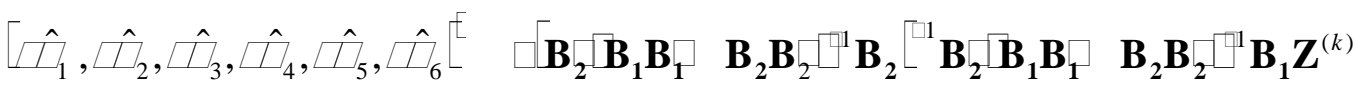

which we can write as follows:

$$
\begin{aligned}
& \Pi_{\mathbf{1}} \mathbf{Z}^{(\mathbf{k})}\left(\begin{array}{c}
\varpi_{1,1} \\
\square_{1,2} \\
\vdots \\
\square_{n, 1} \\
\square_{n, 2} \\
\square_{1,1} \\
\square_{1,2} \\
\vdots \\
\varpi_{n, 1} \\
\square_{n, 2}
\end{array}\right)
\end{aligned}
$$

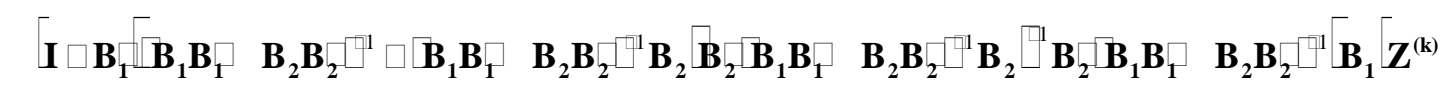

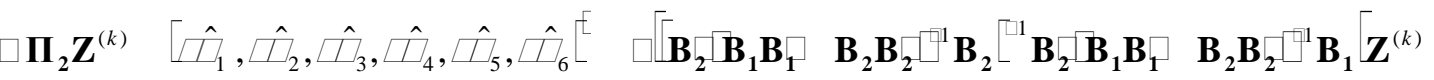




\section{Bibliography}

[1] ÚZIS: Aktuální informace č. 14 - Kardiochirurgické operace (http://www.uzis.cz/cz/archiv04/14_04.pdf)

[2] Takami, Y., Ina, H.: A simple Method to Determine Anastomotic Quality of Coronary Artery Bypass Grafting in the Operating Room. Cardiovascular Surgery, Vol. 9, No. 5, pp. 499-503

[3] Takami, Y., Ina, H.: Relation of Intraoperative Flow Measurement With Postoperative Quantitative Angiographic Assessment of Coronary Artery Bypass Grafting. Ann Thorac Surg 2001, 72:1270-4

[4] D’Anconna, G., Karamanoukian, H. L., Bergsland, J.: Is Intraoperative measurement of coronary blood flow a good predictor of graft patency? European Journal of Cardiothoracic Surgery 20 (2001), pp. 1075-1076

[5] Shin, H. et al.: Intraoperative Assessment of Coronary Artery Bypass Graft: TransitTime Flowmetry Versus Angiography. Ann Thorac Surg 2001, 72:1562-5

[6] Louagie, Y. et al.: Pulsed Doppler Intraoperative Flow Assessment and Midterm Coronary Graft Patency. Ann Thorac Surg 1998, 66:1282-8

[7] Louagie, Y., Brockmann, C., Gurné, O., Jamart, J.: Intraoperative Flow Measurements: Predictive Value for Postoperative Angiographic Follow-Up. In.: Intraoperative Graft Patenty Verification in Cardiac and Vascular Surgery (ed. G. D'Ancona). Futura Publishing, Armonk (NY) 2001

[8] Milnor, W. R.: Hemodynamics. Williams\&Wilkins, Baltimore (USA) 1998

[9] Hata, M. et al.: Midterm Results of Coronary Artery Bypass Graft Surgery With Internal Thoracic Artery Under Low Free-Flow Conditions. Ann Thorac Surg 2004, 78:477-80

[10] Pagni, S. et al.: Factors Affecting Internal Mammary Artery Graft Survival: How Is Competitive Flow from a Patent Native Coronary Vessel a Risc Factor? Journal of Surgical Research 71 (1997), pp. 172-178

[11] Sabik, J. F. et al.: Does Competitive Flow Reduce Internal Thoracic Artery Graft Patency? Ann Thorac Surg 2003, 76:1490-7

[12] Pagni, S., Storey, J. et al.: ITA versus SVG: a comparison of instantaneous pressure and flow dynamics during competitive flow. European Journal of Cardio-thoracic Surgery 11 (1997), pp. 1086-1092

[13] Lausten, J.: Transit-Time Flow Measurement: Principles and Clinical Applications. In.: Intraoperative Graft Patenty Verification in Cardiac and Vascular Surgery (ed. G. D’Ancona). Futura Publishing, Armonk (NY) 2001

[14] Kubáček, L., Kubáčková, L.: Statistika a metrologie. VUP Olomouc 2000

[15] Kubáček, L., Kubáčková, L., Volaufová, J.: Statistical Models with Linear Structures. Veda, Bratislava 1995. 